\title{
Pancreatic cancer modeling using retrograde viral vector delivery and in vivo CRISPR/Cas9-mediated somatic genome editing
}

\author{
Shin-Heng Chiou, ${ }^{1,13}$ Ian P. Winters, ${ }^{1,13}$ Jing Wang, ${ }^{2,3}$ Santiago Naranjo, ${ }^{1}$ Crissy Dudgeon, ${ }^{4,5,6}$ \\ Fiona B. Tamburini, ${ }^{1}$ Jennifer J. Brady, ${ }^{1}$ Dian Yang, ${ }^{7}$ Barbara M. Grüner, ${ }^{1}$ Chen-Hua Chuang, ${ }^{1}$ \\ Deborah R. Caswell, ${ }^{7}$ Hong Zeng, ${ }^{8,9}$ Pauline Chu, ${ }^{10}$ Grace E. Kim, ${ }^{11}$ Darren R. Carpizo, ${ }^{4,5,6}$ \\ Seung K. Kim, ${ }^{2,3}$ and Monte M. Winslow ${ }^{1,7,8,12}$ \\ ${ }^{1}$ Department of Genetics, Stanford University School of Medicine, Stanford, California 94305, USA; ${ }^{2}$ Department of \\ Developmental Biology, Stanford University School of Medicine, Stanford, California 94305, USA; ${ }^{3}$ Howard Hughes Medical \\ Institute, Stanford University School of Medicine, Stanford, California 94305, USA; ${ }^{4}$ Rutgers Cancer Institute of New Jersey, New \\ Brunswick, New Jersey 08903, USA; ${ }^{5}$ Department of Surgery, Rutgers Robert Wood Johnson University Medical School, New \\ Brunswick, New Jersey 08903, USA; ${ }^{6}$ Department of Pharmacology, Rutgers Robert Wood Johnson University Medical School, \\ New Brunswick, New Jersey 08903, USA; ${ }^{7}$ Cancer Biology Program, Stanford University School of Medicine, Stanford, California \\ 94305, USA; ${ }^{8}$ Stanford Cancer Institute, Stanford University School of Medicine, Stanford, California 94305, USA; ${ }^{9}$ Transgenic, \\ Knockout, and Tumor Model Center, Stanford University School of Medicine, Stanford, California 94305, USA; ${ }^{10}$ Department of \\ Comparative Medicine, Stanford University School of Medicine, Stanford, California 94305, USA; ${ }^{11}$ Department of Pathology, \\ University of California at San Francisco, San Francisco, California 94143, USA; ${ }^{12}$ Department of Pathology, Stanford University \\ School of Medicine, Stanford, California 94305, USA
}

Pancreatic ductal adenocarcinoma (PDAC) is a genomically diverse, prevalent, and almost invariably fatal malignancy. Although conventional genetically engineered mouse models of human PDAC have been instrumental in understanding pancreatic cancer development, these models are much too labor-intensive, expensive, and slow to perform the extensive molecular analyses needed to adequately understand this disease. Here we demonstrate that retrograde pancreatic ductal injection of either adenoviral-Cre or lentiviral-Cre vectors allows titratable initiation of pancreatic neoplasias that progress into invasive and metastatic PDAC. To enable in vivo CRISPR/Cas9-mediated gene inactivation in the pancreas, we generated a Cre-regulated $\mathrm{Cas} 9$ allele and lentiviral vectors that express Cre and a single-guide RNA. CRISPR-mediated targeting of $L k b 1$ in combination with oncogenic Kras expression led to selection for inactivating genomic alterations, absence of Lkb1 protein, and rapid tumor growth that phenocopied Cre-mediated genetic deletion of $L k b 1$. This method will transform our ability to rapidly interrogate gene function during the development of this recalcitrant cancer.

[Keywords: CRISPR; genome editing; mouse model; pancreatic cancer]

Supplemental material is available for this article.

Received April 29, 2015; revised version accepted June 19, 2015.

Pancreatic ductal adenocarcinoma (PDAC) is an almost uniformly lethal tumor type that is projected to become the second leading cancer killer in the United States by 2030 (Rahib et al. 2014). PDAC patients have a 5-year sur-

\footnotetext{
${ }^{13}$ These authors contributed equally to this work. Corresponding author: mwinslow@stanford.edu Article published online ahead of print. Article and publication date are online at http://www.genesdev.org/cgi/doi/10.1101/gad.264861.115.
}

vival rate of $\sim 5 \%$, underscoring the need for novel approaches to accelerate the molecular characterization of this disease. Although high-prevalence mutations have been identified in pancreatic cancer, these tumors also

(C) 2015 Chiou et al. This article is distributed exclusively by Cold Spring Harbor Laboratory Press for the first six months after the full-issue publication date (see http://genesdev.cshlp.org/site/misc/terms.xhtml). After six months, it is available under a Creative Commons License (Attribution-NonCommercial 4.0 International), as described at http:// creativecommons.org/licenses/by-nc/4.0/. 
incur low-frequency mutations and genomic alterations, interact with their extensive and complex stromal environment, and undergo poorly characterized changes in their gene expression programs (Biankin et al. 2012; Waddell et al. 2015). Despite the potential importance of these molecular and cellular changes, very little is known about how these alterations contribute to the development of metastatic and therapy-refractory PDAC. Given the inability to test gene function in human cancers in vivo, genetically engineered mouse models represent tractable and biologically relevant systems with which to interrogate the molecular determinants of each stage of pancreatic cancer development.

Identification of the mutations that drive the development of human pancreatic cancer combined with the ability to alter gene function in mice has enabled the development of genetically engineered murine PDAC models. Transgenic expression of Cre-recombinase in pancreatic cells of loxP-Stop-loxP (LSL) Kras ${ }^{\text {G12D }}$ knock-in mice $\left(\mathrm{Kras}^{\mathrm{LSL}-\mathrm{G} 12 \mathrm{D} /+}\right)$ results in deletion of the transcriptio$\mathrm{nal} /$ translational Stop element, expression of oncogenic Kras ${ }^{\mathrm{G} 12 D}$, and development of lesions that closely resemble early stage human pancreatic intraepithelial neoplasms (PanINs) (Hingorani et al. 2003). Concomitant expression of a point mutant p53 allele, deletion of p53, deletion of $C d k n 2 a$, and/or deletion of Smad4 allow(s) for the development of invasive and metastatic PDAC /Aguirre et al. 2003; Hingorani et al. 2005; Bardeesy et al. 2006a,b; Gidekel Friedlander et al. 2009; Whittle et al. 2015).

These in vivo models have been instrumental in our understanding of the genetic determinants of cancer progression as well as the functional interactions of neoplastic cells with the immune system and stromal environment. However, using conventional genetically engineered autochthonous mouse models of PDAC to interrogate gene function is complicated by inherent practical and biological limitations of these systems. Existing mouse models typically fail to model the adult onset of pancreatic cancer and induce genomic alteration in nearly every cell in the pancreas. In the decade since the first genetically engineered PDAC models were developed, few technical advances have been made, and generating the mice required to investigate a gene of interest in the established PDAC models remains a time-consuming and costly endeavor (Aguirre et al. 2003; Hingorani et al. 2003, 2005; Saborowski et al. 2014).

Systems that enable in vivo functional interrogation of genes in pancreatic cancer without the financial and temporal cost of generating new mouse alleles and incorporating them into increasingly complex mouse models could have an extremely broad impact on pancreatic cancer research. To functionally investigate the molecular changes that drive each step of pancreatic cancer development, it would be desirable to have a system in which the timing of tumor initiation and the number of lesions that form in the adult pancreas can be controlled, the number of germline-encoded alleles is minimized, and genes of interest can be eliminated without having to generate a conditional allele and breed it into a complex genetically engineered mouse model.
Here we describe methods for the direct delivery of viral vectors to the pancreas and transgenic mouse lines to allow CRISPR/Cas9-mediated genomic alterations in pancreatic cells in vivo. These systems allow titratable initiation of pancreatic tumors in adult mice and functional interrogation of candidate genes in pancreatic cancer in vivo.

\section{Results}

Retrograde pancreatic ductal injection of adenoviral-Cre (Adeno-Cre) leads to infection of pancreatic cells

Current genetically engineered mouse models of human PDAC are limited by their complete reliance on germline conditional and transgenic Cre alleles that lead to widespread recombination in the majority of the epithelial cells, most often within the embryonic pancreas. Since viral-Cre infection of the lung, prostate, bladder, and muscle can initiate cancer in adult tissues (Jackson et al. 2001; Kirsch et al. 2007; Puzio-Kuter et al. 2009; Cho et al. 2014), we considered how best to deliver viral-Cre to the pancreas without infecting other cells within the peritoneal cavity. To specifically initiate recombination in cells within the adult pancreas while keeping the virus contained within the target organ, we used retrograde pancreatic ductal injection. In this procedure, the virus is directly injected into the pancreas through the common bile duct that drains the biliary and pancreatic fluid into the duodenum (Fig. 1A,B; Supplemental Movie 1; Taniguchi et al. 2003; Wang et al. 2006). Injection of adenoviralCMV-Cre (Ad-Cre) into Cre reporter mice (R26 $6^{\text {LSL-Tom }}$ ) (Madisen et al. 2010) led to sporadic labeling of mostly acinar cells within the pancreas, with no detectable infection of cells within the liver, intestine, or any other organs outside the pancreas (Fig. 1C; Supplemental Fig. 1A-C; data not shown).

Retrograde ductal injection of Adeno-Cre and lentiviral-Cre (Lenti-Cre) initiates pancreatic tumors

Retrograde ductal injection of $\mathrm{KraS}^{\mathrm{LSL}-\mathrm{G} 12 \mathrm{D} /{ }^{+}}{ }_{;} \mathrm{R}_{26} 6^{\mathrm{LSL}-\mathrm{Tom}}$ $(K T)$ mice with Ad-Cre led to acinar-to-ductal metaplasia (ADM) and the formation of hundreds of Tomato ${ }^{\text {positive }}$ PanIN lesions, which expressed cytokeratin 19 (CK19) (Fig. 1D,E). These PanIN lesions had abundant apical mucin with small oval nuclei, and none of the lesions developed beyond PanIN1 over a 6-mo period (Fig. 1D-F; Supplemental Table 1; Supplemental Fig. 1E,F).

To determine whether retrograde ductal injection could initiate cancer when oncogenic Kras ${ }^{\mathrm{G} 12 \mathrm{D}}$ expression was combined with loss of the p53 tumor suppressor, we injected Ad-Cre into the pancreata of $\mathrm{Kras}^{\mathrm{LSL}-\mathrm{G12D} /{ }^{+}}$; p53 ${ }^{\text {flox } / \text { flox }} ; R 26^{\text {LSL-Tom }}(K P T)$ mice (Fig. 1G-J). At early time points, KPT mice infected with Ad-Cre already harbored numerous ADMs and PanIN lesions of both early and advanced grades (Fig. 1G,H; Supplemental Fig. 2A-E). At later time points, these mice became 

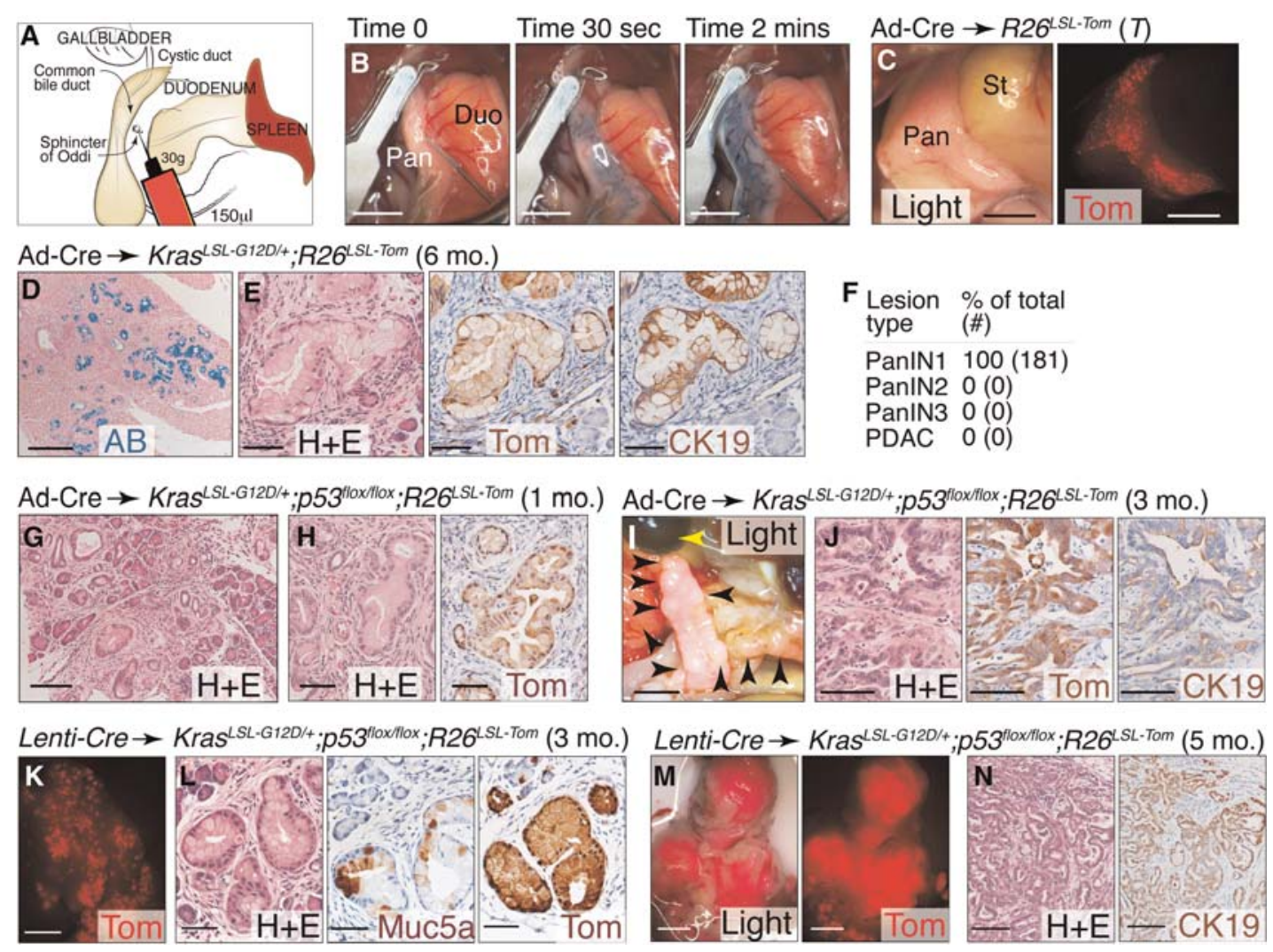

Figure 1. Pancreatic retrograde ductal injection of Adeno-Cre or Lenti-Cre induces widespread recombination and initiates the development of PanINs and ductal adenocarcinoma. $(A)$ Diagram of the pancreatic retrograde ductal injection procedure. $(B)$ Images of retrograde ductal injection of a blue dye highlight the distribution of the injected fluid and the expansion of the injected tissue. Bars, $3 \mathrm{~mm}$. (Pan) Pancreas; (Duo) duodenum. $(C)$ Retrograde ductal Ad-Cre-injected R26 $6^{L S L-T o m}(T)$ mice $(n=3)$ have widespread infection of the pancreas. Mice were analyzed $7 \mathrm{~d}$ after infection. Bars, $3 \mathrm{~mm}$. (Pan) Pancreas; $(\mathrm{St})$ stomach. $(D, E)$ Retrograde ductal Ad-Cre-injected Kras ${ }^{L S L-G 12 D /+}$; $R 26^{L S L-T o m}(K T)$ mice $(n=4)$ develop PanINs (6-7 mo after tumor initiation). Bars: $D, 200 \mu \mathrm{m} ; E, 50 \mu \mathrm{m}$. (AB) Alcian blue. (F) Quantification of pancreatic lesions in Ad-Cre-infected $K T$ mice 6-7 mo after tumor initiation. $(G, H)$ At early time points, retrograde ductal Ad-Cre-

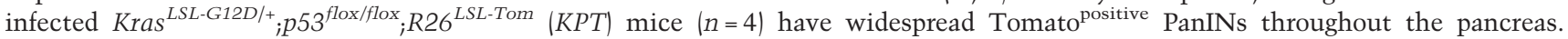
Bars: $G, 100 \mu \mathrm{m} ; H, 50 \mu \mathrm{m}$. $(I, J)$ Retrograde ductal Ad-Cre-injected $K P T$ mice $(n=19)$ develop PDAC. The yellow arrowhead indicates the enlarged gallbladder consistent with biliary obstruction. Black arrowheads indicate tumors. Bars: $I, 3 \mathrm{~mm} ; J, 50 \mu \mathrm{m} .(K, L)$ Retrograde ductal Lenti-Cre-injected KPT mice develop Tomato ${ }^{\text {positive }}$ PanIN lesions (3 mo after infection). Bars: $K, 2$ mm; $L, 50 \mu$ m. (M,N) Retrograde ductal Lenti-Cre-injected KPT mice develop Tomato ${ }^{\text {positive }}$ PDAC. A moderately differentiated CK19 ${ }^{\text {positive }}$ PDAC is shown. Bars: $M, 3 \mathrm{~mm} ; N, 100 \mu \mathrm{m}$.

cachectic and jaundiced and displayed poor grooming due to the development of multifocal pancreatic tumors (Fig. 1I,J; Supplemental Fig. 2F,G; Supplemental Table 1).

KPT mice infected with Ad-Cre $\left(1 \times 10^{8}\right.$ to $\left.5 \times 10^{6} \mathrm{IU}\right)$ lived between 2 and 6 mo depending on the viral titer and developed invasive ductal adenocarcinomas similar to those observed in the conventional transgenic-Crebased models (Fig. 1I,J; Supplemental Fig. 2F,H; Bardeesy et al. 2006a). Tomato ${ }^{\text {positive }}$, CK19 ${ }^{\text {positive }}$ ductal adenocarcinomas were characterized by the formation of glandular structures. These tumors often had extensive, collagenrich extracellular matrix deposition and abundant, Tomato $^{\text {negative }}$ desmoplastic stromal responses (Fig. 1J; Supplemental Fig. 2F).

Ad-Cre infection of mice with a conditional point mutant p53 allele (Kras ${ }^{L S L-G 12 D /+} ;$ p53 ${ }^{L S L-R 172 H / f l o x} ;$
R26 $6^{\text {LSL-Tom }}$ also initiated PDAC with overall similar histological features (Supplemental Fig. 2I). Finally, neither Ad-Cre infection of $p 53^{\text {flox } / f l o x}$; $R 26^{\text {LSL-Tom }}$ mice, which lack the oncogenic Kras allele, nor control adenovirus (adenoviral-CMV-FLPo [Ad-FLPo]) infection of KPT mice resulted in any pancreatic lesions (Supplemental Table 1; Supplemental Fig. 2G; data not shown).

To specifically target acinar cells in the pancreas, we generated an adenoviral vector that contains a $2.3-\mathrm{kb}$ acinar cell-specific Ptf1a enhancer and Ela1 minimal promoter driving Cre (Ad-Ptf1a-Cre). Retrograde ductal injection of $R 26^{L S L-T o m}$ mice with Ad-Ptf1a-Cre documented the specificity of this promoter for acinar cells (Supplemental Fig. 3A-D). KPT mice infected with Ad-Ptf1aCre developed PanINs and invasive metastatic PDAC, consistent with our results using Ad-CMV-Cre and consistent with acinar cells within the adult pancreas being 
a cell of origin for PDAC (Supplemental Fig. 3E-H; Guerra et al. 2007).

The use of lentiviral vectors to initiate pancreatic cancer would offer a modular system to rapidly alter genes in developing tumors due to their stable genomic integration. Retrograde ductal injection of Lenti-Cre into R26 $6^{\text {LSL-Tom }}$ mice led to labeling of mostly acinar cells (Supplemental Fig. 4A-C). KT mice infected with LentiCre developed exclusively low-grade PanINs (Supplemental Fig. 4D). KPT mice infected with Lenti-Cre developed PanINs and PDAC 2-6 mo after tumor initiation (Fig. 1KN; Supplemental Fig. 2J,K). Viral-Cre-initiated pancreatic tumors ranged from well differentiated to undifferentiated, and the spectrum of differentiation within primary tumors and metastases was similar for Adeno-Cre and LentiCre (Fig. 1J,N; Supplemental Fig. 2F,J; Supplemental Table 1 ; data not shown). In many cases, Ad-Cre-induced and Lenti-Cre-induced tumors developed in the head of the pancreas and were accompanied by evidence of gallbladder obstruction and jaundice, a frequent end-stage complication of pancreatic cancer in patients (Supplemental Table 1; Supplemental Fig. 2G; Kruse 2010; Boulay and Parepally 2014).

Chronic pancreatitis is associated with an increased incidence of PDAC in humans, and experimental evidence from mouse models suggests that adult cells may be refractory to transformation in the absence of pancreatic inflammation (Guerra et al. 2007; Raimondi et al. 2010). Therefore, we assessed whether retrograde ductal viral infection induces pancreatitis. Both Ad-Cre and LentiCre infection induced acute pancreatitis with morphological evidence of ADM and focal replacement of acinar cells by infiltrating mononuclear cells (Supplemental
Figs. $1 \mathrm{C}, \mathrm{D}, 4 \mathrm{C})$. The induction of pancreatitis is consistent with the efficient tumor initiation observed in adult animals following retrograde ductal injection of viral-Cre.

Use of virally induced PDAC to investigate the kinetics and genetics of metastatic progression

A key attribute of human pancreatic cancer is its invasive growth and propensity for widespread metastasis. We used the bright Tomato reporter to mark neoplastic cells in virally induced tumors, thereby allowing for quantification of disseminated tumor cells (DTCs) in the peritoneal cavity and micrometastases and macrometastases in $K T$ and KPT mice (Fig. 2A-G). Tomato ${ }^{\text {positive }}$ DTCs could also be easily identified in the peritoneal cavity of some late time point KPT mice (Fig. 2A,D,G). KPT mice developed widespread metastases in the liver, diaphragm, lung, and mesenteric lymph nodes, all of which are prevalent sites for human PDAC metastasis (Fig. 2B-G; Supplemental Fig. 5; Supplemental Table 1; Yachida and Iacobuzio-Donahue 2009|. Despite the high total tumor burden in most Ad-Cre-infected and Lenti-Cre-infected KPT mice, the number of DTCs in the peritoneal cavity was quite variable, most consistent with the notion that the primary tumors must progress to a cellular state capable of dissemination (Fig. 2D,G). The absence of DTCs and metastases in KT mice was consistent with the presence of only homogeneously low-grade PanIN lesions and suggests that other engineered tumor suppressor alterations or spontaneous cell state alterations are a prerequisite for neoplastic cell dissemination.
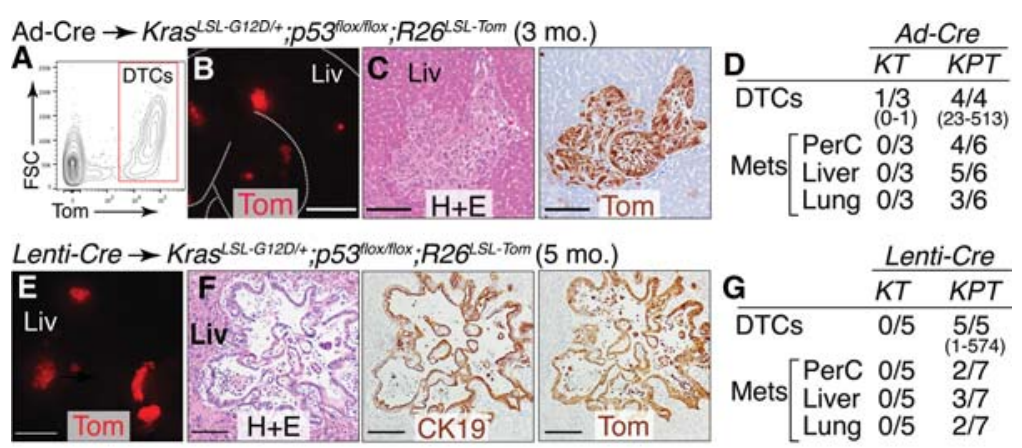

\begin{tabular}{|c|c|c|c|}
\hline \multirow{2}{*}{\multicolumn{2}{|c|}{ G }} & \multicolumn{2}{|c|}{ Lenti-Cre } \\
\hline & & $K T$ & $K P T$ \\
\hline$\overline{\mathrm{DTC}}$ & & $0 / 5$ & $\begin{array}{c}5 / 5 \\
(1-574)\end{array}$ \\
\hline Mets & {$\left[\begin{array}{l}\text { PerC } \\
\text { Liver } \\
\text { Lung }\end{array}\right.$} & $\begin{array}{l}0 / 5 \\
0 / 5 \\
0 / 5\end{array}$ & $\begin{array}{l}2 / 7 \\
3 / 7 \\
2 / 7\end{array}$ \\
\hline
\end{tabular}
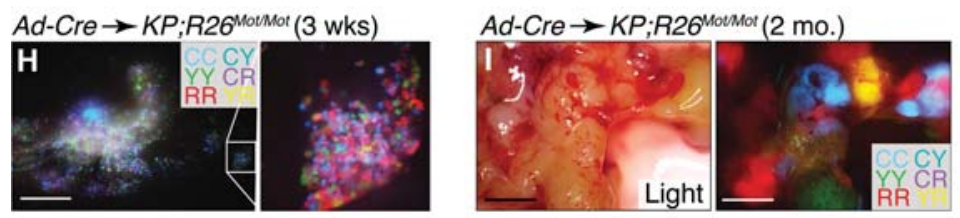

Lenti-Cre $\rightarrow K P ; R 26^{\text {MotMot }}(4 \mathrm{mo}$.)

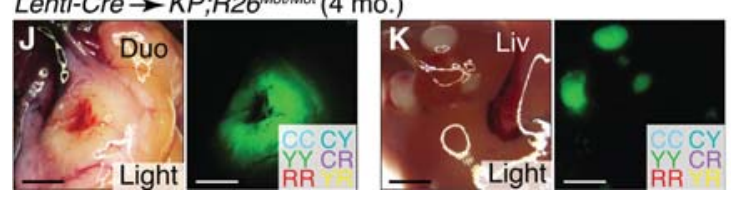

Figure 2. Diverse expansion potential and metastatic ability of viral-Cre-initiated PDAC. (A) DTCs can be detected in the peritoneal cavity of Ad-Cre-infected KPT mice. Viable (DAPI $\left.{ }^{\text {negative }}\right)$, lineage-negative (CD31, CD45, Ter119, F4/80) $)^{\text {negative }}$ cells are shown. $(B, C)$ Retrograde ductal Ad-Cre tumor initiation in KPT mice leads to widespread metastasis. A dotted outline of liver (Liv) lobes is shown in $B$. (C) Representative histology of liver metastasis. Bars: $B, 3$ mm; $C, 100 \mu \mathrm{m}$. (D) Quantification of DTCs and metastases in Ad-Cre-infected KT $\left(10^{9}\right.$ IU Ad-Cre, $\left.6 \mathrm{mo}\right)$ and KPT $\left(10^{7}-10^{8} \mathrm{IU}\right.$ Ad-Cre, 3-7 mol mice. Data represent the number of mice with the indicated lesion/total number of mice of that genotype. For DTCs, the range of the total DTC number is shown in parentheses. "PerC" indicates metastases in the peritoneal cavity. $(E, F)$ KPT mice with Lenti-Cre-induced PDAC can develop multifocal, multiorgan metastatic disease. (Liv) Liver. Bars: E, 2 mm; F, $100 \mu \mathrm{m} .(G)$ Quantification of DTCs and metastases in LentiCre-infected KT and KPT mice. Data are represented as in $D$. $(H-K)$ Injection of Ad-Cre $(H, I)$ or Lenti-Cre $(J, K)$ into $\operatorname{Kras}^{\text {LSL-G12D/+}}{ }^{2}$ p53 $^{\text {flox } / f l o x}$; $R 26^{\text {Mot/Mot }}\left(K P ; R 26^{\text {Mot/Mot }}\right)$ mice initiates diverse color labeling of early lesions $(H)$, few of which progress into tumors $(I)$. $(J, K)$ A single, clonally derived, late time point primary tumor $(J)$ seeded multiple liver metastases $(K)$. (Duo) Duodenum; (Liv) liver. Bars, $3 \mathrm{~mm}$. 
Identifying the clonal origin of PDACs and determining tumor-metastasis relationships

Conventional transgenic Cre-driven and Cre(ER)-driven PDAC models lack the ability to distinguish between a tumor mass derived from a single transformed cell and a mass of collision tumors. To assess the clonality of viral Cre-initiated tumors, we recently generated a multicolor Cre reporter in which recombination leads to the expression of RFP, CFP, or YFP (R26 $\left.6^{\text {Motley }}\right)$ (Caswell et al. 2014). Retrograde ductal Ad-Cre infection of $R 26^{\text {Motley/Motley }}$ homozygous mice generated cells with each of the six color combinations (Supplemental Fig. 6A). Infection of Kras $^{\text {LSL-G12D/+}}{ }_{;}$p5 $^{\text {flox/flox }}{ }_{;} R_{2} 6^{\text {Motley/Motley }}$ (KP;R26 $6^{\text {Mot/Mot }}$ ) mice generated large numbers of ADMs and PanINs, a relatively small number of which progressed into individual tumors within the pancreatic cancer mass (Fig. 2H,I; Supplemental Fig. 6B-E). Both Ad-Cre-infected and LentiCre-infected $K P ; R 26^{\text {Mot/Mot }}$ mice developed clonally derived monochromatic tumors (Fig. 2J; Supplemental Fig. 6F,G). Some viral-Cre-infected $K P ; R 26^{\text {Mot } / M o t}$ mice progressed to have one dominant tumor, and, whenever metastases were present, they were the same color as the largest primary PDAC (Fig. 2J,K; Supplemental Fig. $6 \mathrm{E}-\mathrm{G})$.

Generation and characterization of Cre-regulated Cas9 mice and lentiviral vectors for single-guide RNA (sgRNA) and Cre expression

The ability to induce targeted genomic deletions in pancreatic cancer in vivo would facilitate the investigation of all aspects of pancreatic tumorigenesis. To enable in vivo somatic genomic editing in mouse models of human cancer, we generated single insertion transgenic mice through site-directed integration of a $L S L$-Cas9 cassette into the H11 locus $\left(H 11^{\text {LSL-Cas }}\right.$ ) (Fig. 3A,B; Supplemental Fig. 7A; Tasic et al. 2011). Expression of Cre in fibroblasts from $H 11^{\text {LSL-Cas } 9}$ mice induced recombination of the Stop cassette and led to expression of Cas 9 RNA and protein (Fig. 3C,D; data not shown). To characterize the potential for Cas9 expression from the H11 ${ }^{L S L \text {-Cas } 9}$ allele in vivo, we intercrossed $H 11^{L S L-C a s 9}$ and $C M V$-Cre deleter mice to generate an $H 11^{\text {Cas } 9}$ allele. $H 11^{\text {Cas } 9}$ mice had widespread expression of Cas9 across all organs tested (Supplemental Fig. 8).

To deliver a synthetic sgRNA and Cre into pancreatic cells, we generated dual promoter lentiviral vectors that contain a U6-driven sgRNA and also express Cre (Supplemental Fig. 7B). Given the frequent down-regulation and genomic loss of LKB1/STK11 in human PDAC (Morton et al. 2010; Waddell et al. 2015), the predisposition of Peutz-Jeghers syndrome patients to develop pancreatic tumors (Su et al. 1999), and the impact of Lkb1 inactivation in other pancreatic tumor models (Hezel et al. 2008; Morton et al. 2010; Lo et al. 2012), we chose $L k b 1$ as an initial target for CRISPR/Cas9-mediated somatic alteration in the adult pancreas. To assess the impact of $L k b 1$ loss on pancreatic cancer initiation and growth, we generated and screened Lenti-sgRNA/Cre vectors targeting $L k b 1$

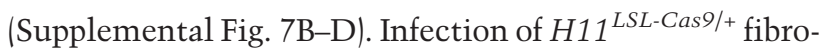
blasts with Lenti-sgLkb1/Cre led to the formation of insertions and deletions (indels) in $L k b 1$ at the targeted location, thus validating both our lentiviral sgRNA vector and the H11 ${ }^{\text {LSL-Cas } 9}$ transgenic allele (Fig. 3E; Supplemental Fig. 7E).

Somatic genome engineering enables rapid generation of genetically defined pancreatic cancer mouse models

To assess the impact of Lkb1 deletion on pancreatic cancer, we performed retrograde ductal injections of $\mathrm{KT} ; \mathrm{H} 11^{\text {LSL- }}$

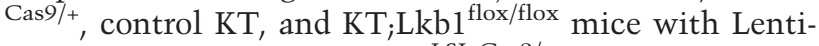
sgLkb1/Cre as well as $\mathrm{KT}_{;} \mathrm{H} 11^{\mathrm{LSL}-\mathrm{Cas} 9 /+}$ mice with lentiviral vectors containing negative control sgRNAs. Control $\mathrm{KT}$ mice infected with Lenti-sgLkb1/Cre as well as $\mathrm{KT}$; $\mathrm{H} 11^{\text {LSL-Cas9/+ }}$ mice infected with a Lenti-sgRNA/Cre vector containing either a nontargeting sgRNA (sgNT) or an sgRNA targeting an inert region of the genome (sgNeo) formed only rare Tomato ${ }^{\text {positive }}$ lesions (Fig. 3F-H). Both Lenti-sgLkb1/Cre-infected $\mathrm{KT} ; \mathrm{H}_{1} 1^{\text {LSL-Cas9/+ }}$ and $\mathrm{KT}$; $\mathrm{Lkb1}^{\text {flox/flox }}$ mice had extensive tumor growth as early as 2 mo after tumor initiation (Fig. 3I,J, respectively).

All three groups of negative control mice developed only rare PanIN lesions within almost completely normal pancreata (Fig. 4A-C; Supplemental Fig. 9A; data not shown). The tumors that formed in Lenti-sgLkb1/Cre-infected KT;H11 $1^{\text {LSL-Cas } /++}$ mice were cystic lesions comprised of Tomato $^{\text {positive }}, \mathrm{CK} 19^{\text {positive }}$ tall cuboidal to columnar epithelial cells with otherwise bland cytological features (Fig. 4D; Supplemental Fig. 9B). Some smaller lesions also contained high levels of mucin (Supplemental Fig. 9B). Importantly, these features were histologically indistinguishable from those found in $K T ; L k b 1^{\text {flox/flox }}$ mice infected with Lenti-sgLkb1/Cre (Fig. 4E; Supplemental Fig. 9C). Lenti-sgLkb1/Cre-infected KT;H11 ${ }^{L S L-C a s 9 /+}$ mice also had a substantially higher tumor burden when compared with all three groups of negative control mice (Fig. 4F).

FACS-sorted Tomato ${ }^{\text {positive }}$ cells from Lenti-sgLkb1/ Cre-infected KT;H11 ${ }^{\text {LSL-Cas } 9 /+}$ mice had recombined the H11 ${ }^{\text {LSL-Cas } 9}$ allele and expressed Cas9 RNA and protein (Supplemental Fig. 9D,E; data not shown). Diverse indels at the targeted $L k b 1$ locus were specifically detected in the neoplastic cells from these tumors (Fig. 5A,B; Supplemental Fig. 9F). The indels were all frameshift mutations or large exon deletions that included the splice acceptor or donor regions of $L k b 1$ exon 6, further supporting the strong selective advantage of $L k b 1$ inactivation (Fig. 5B, C). Consistent with the presence of CRISPR/Cas9-induced frameshift mutations and large deletions, Lkb1 protein was absent from most neoplastic cells in the LentisgLkb1/Cre-induced tumors in KT;H11 ${ }^{\text {LSL-Cas } /++}$ mice (Fig. 5D,E; Supplemental Fig. 9G).

\section{Discussion}

The development of systems that accelerate our ability to investigate pancreatic carcinogenesis at the molecular 
A

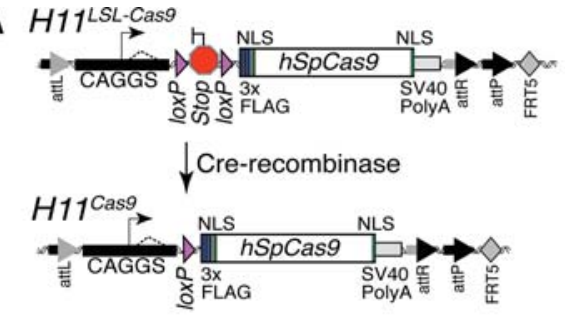

B

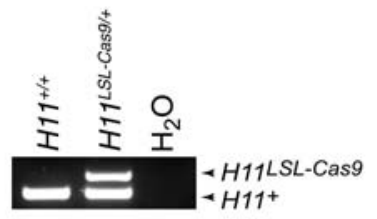

C

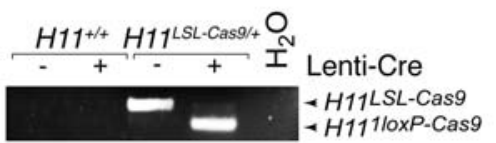

D

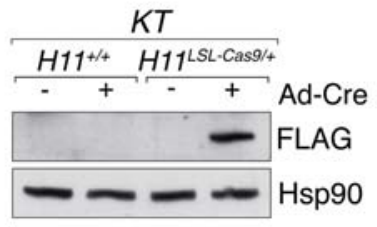

E

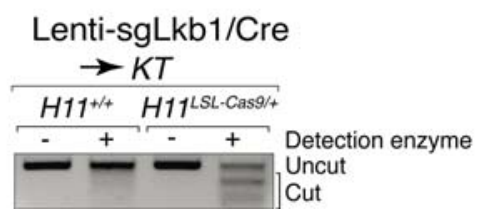

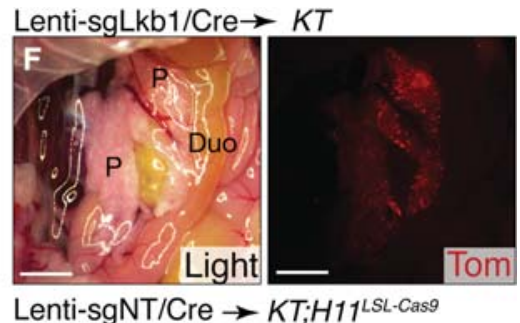

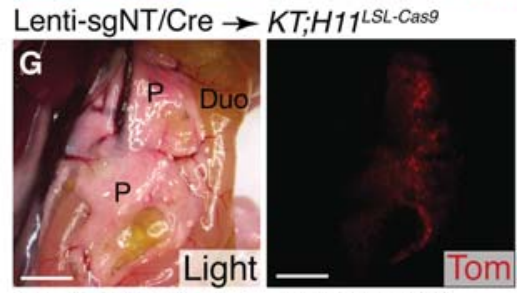

Lenti-sgNeo/Cre $\rightarrow K T ;$ H11 $^{\text {LSL-Cas } 9}$

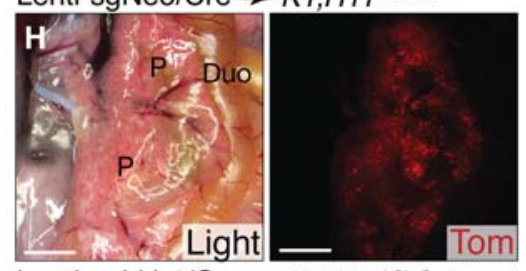

Lenti-sgLkb1/Cre $\rightarrow K T ; H 11^{\text {LSL-Cas9 }}$

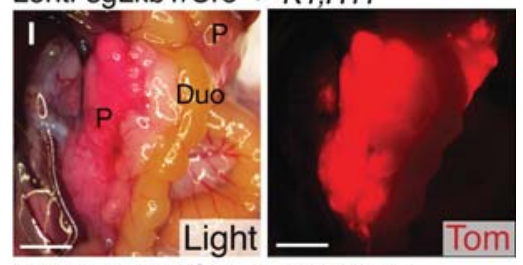

Lenti-sgLkb1/Cre $\rightarrow K T ; L k b 1^{1 / 4}$

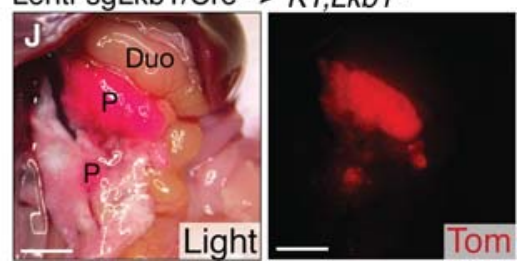

Figure 3. CRISPR/Cas9 enables in vivo genetic alteration in pancreatic cancer. $(A)$ Schematic of the Cre-regulated Cas9 allele. (CAGGS) Cytomegalovirus immediate-early enhancer/chicken $\beta$-actin promoter; (hSpCas9) human codon-optimized Streptococcus pyogenes Cas9; (NLS) nuclear localization signal. $(B)$ PCR genotyping of H11 ${ }^{L S L-C a s 9}$ mice. (C) Efficient Cre-mediated recombination of the $L S L$ cassette into a single LoxP site (1LoxP) in H11 ${ }^{\text {LSL-Cas9 }}$ cells in vitro. These primers do not recognize the $H 11^{\text {wt }}$ locus. (D) Cre-regulated expression of Flag-tagged Cas9 protein in fibroblasts derived from $H 11^{\text {LSL-Cas } 9}$ mice. Hsp90 shows equal loading. $(E)$ Genomic cleavage detection assay for the targeted region of $L k b 1$ in Lenti-sgLkb1/Cre-infected Tomato ${ }^{\text {positive }}$ fibroblasts from $K T$ and $K T ; H 11^{\text {LSL-Cas } 9 /+}$ mice in vitro. $(F-J)$ Representative light and fluorescent images of the pancreata from $K T(n=5), K T ; H 11^{L S L-C a s 9 /+}(n=3)$, and $K T$; $L k b 1^{\text {flox/flox }}(n=3)$ mice 2-3 mo after retrograde ductal injection of Lenti-sgLkb1/Cre as well as from $K T ; H 11^{L S L-C a s 9 /+}$ mice infected with nontargeting Lenti-sgNT/Cre $(n=5)$ or Lenti-sgNeo/Cre, which targets the neomycin resistance gene $(n=2)$. (G) LentisgLkb1/Cre infection of KT;H11 LSL-Cas9/+ mice generates macroscopic Tomato ${ }^{\text {positive }}$ pancreatic tumors. Lenti-sgLkb1/Cre infection of control KT mice $(F)$, Lenti-sgNT/ Cre infection of KT;H11 ${ }^{\text {LSL-Cas } 9 /+}$ mice $(I)$, and Lenti-sgNeo/Cre infection of $K T$; H11 ${ }^{\text {LSL-Cas } 9 /+}$ mice $(J)$ generated only microscopic Tomato ${ }^{\text {positive }}$ areas, while LentisgLkb1/Cre infection of KT;Lkb1 $1^{\text {flox/flox }}$ mice induced macroscopic tumors $(H)$. Bar, $3 \mathrm{~mm}$. (Duo) Duodenum; (P) pancreas. level will be a critical step toward overcoming the dismal rate of successful treatment and low survival rate of patients with this recalcitrant cancer. The ability to induce pancreatic cancer using viral vectors will be instrumental in understanding the mechanisms that sustain tumor growth, lead to metastatic spread, and drive drug resistance. Our CRISPR/Cas9-based model should allow any gene of interest to be inactivated in pancreatic cancer in vivo without the need to generate any new mouse alleles. The method developed in this study could have a profound impact on both basic and translational pancreatic cancer research. Collectively, these methods will enable a more rapid and complete understanding of the molecular regulators of all aspects of pancreatic tumorigenesis and complement the strength of existing genetically engineered models, human patient-derived xenograft models, and studies on human and murine cell lines.

Retrograde ductal injection of Adeno-Cre and Lenti-Cre vectors allows titratable pancreatic tumor initiation in the adult pancreas. This removes the requirement for the transgenic Cre(ER) alleles used in conventional genetically engineered pancreatic cancer models and enables sparse rather than widespread expression of oncogenic Kras and deletion of tumor suppressor genes, more closely recapitulating the initiating events in human PDAC. The inclusion of a fluorescent Cre reporter in transgenic Cre (ER)-induced PDAC models leads to the fluorescent labeling of not only the neoplastic cells but also most of the nontransformed pancreatic epithelial cells, making unequivocal distinction of neoplastic from normal cells difficult.

The ability to induce PDAC in Cre-lox models without having to include a transgenic Cre line will make the molecular investigation of pancreatic cancer more rapid and less expensive (Supplemental Table 2). Additionally, with the ease of generating pancreatic tumors now approaching that of lung tumors (via intranasal or intratracheal injection), comparing the impact of the same genetic alterations on each cancer type should become 

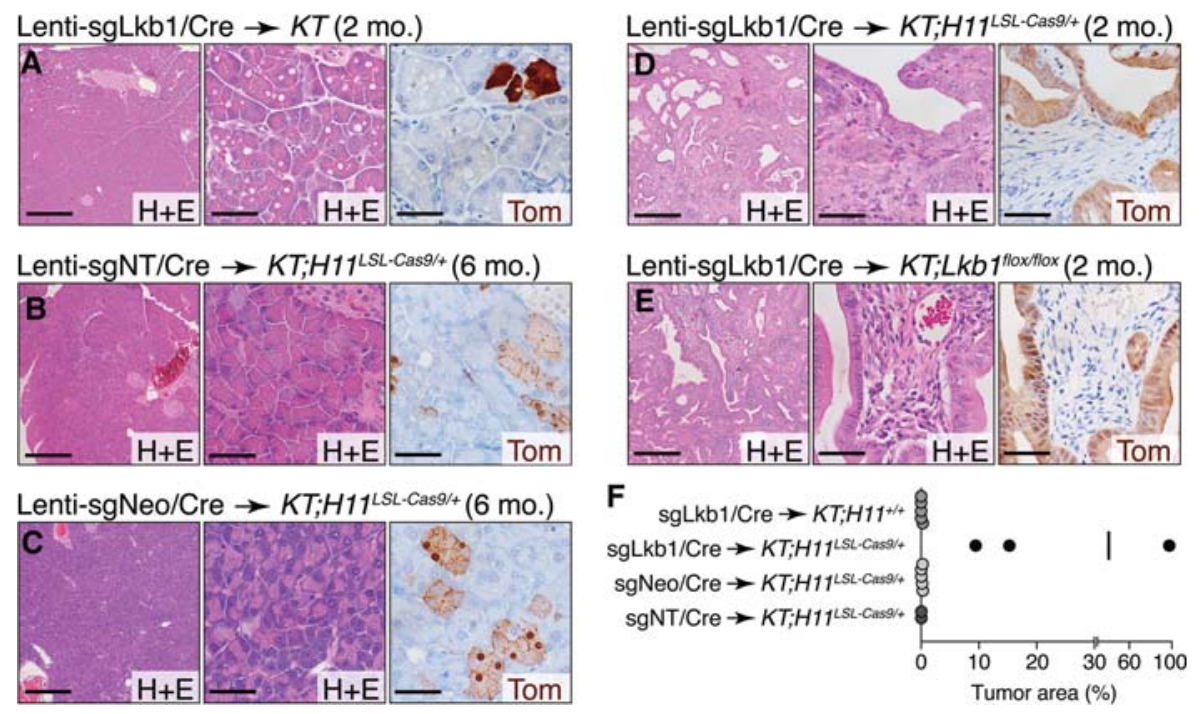

Figure 4. Cas9-mediated targeting of $L k b 1$ in the pancreas promotes tumor growth. $(A)$ Control Lenti-sgLkb1/Cre-infected $K T$ mice $(n=5)$ have small clusters of Tomato ${ }^{\text {positive }}$ cells and very rare ADMs and PanINs. $(B, C) K T ; H 11^{\text {LSL-Cas } /+}$ mice infected with LentisgNT/Cre $(n=5)$ or Lenti-sgNeo/Cre $(n=2)$ do not develop tumors. $(D, E)$ Lenti-sgLkb1/Cre-infected $K T ; H 11^{L S L-C a s 9 /+}$ mice $(n=3)$ develop Tomato ${ }^{\text {positive }}$ cystic tumors that are indistinguishable from the tumors that form in $K T ; L k b 1^{\text {flox/flox }}$ mice $(n=3)$. Bars: $A$ - $E$, left column, $300 \mu \mathrm{m} ; A$-E, right two columns, $30 \mu \mathrm{m}$. $(F)$ Lenti-sgLkb1/Cre-infected $K T ; H 11^{\text {LSL-Cas9/+ }}$ mice have greater tumor area than infected control mice. Each dot represents a mouse, and the bar represents the mean. Note the split scale.

standard practice and will uncover the commonalities and differences between these lethal adenocarcinomas.

PDAC models often generate tumor masses of unknown clonal origin. By incorporating a multicolor fluorescent reporter, we were able to mark individual clonal lesions and identify the relationship between primary tumors and metastases. Unexpectedly, some late time point mice had only one or two large cancers, underscoring the dramatic heterogeneity in expansion potential of pancreatic lesions initiated with identical engineered genetic events. Clonal lineage tracing using diverse fluorescent labeling or lentivirally mediated nucleotide barcoding (Cas- well et al. 2014) may facilitate experiments aimed at understanding early steps in the metastatic process and enable the identification of primary tumors that have and have not gained the ability to seed metastases.

The identity of the cell types capable of giving rise to pancreatic cancer is an area of active investigation and considerable debate (Guerra et al. 2007; Habbe et al. 2008; Gidekel Friedlander et al. 2009; Kopp et al. 2012; Puri et al. 2015). The Adeno-Cre and Lenti-Cre that we used in this study mostly had ubiquitous promoters (CMV and PGK, respectively), which led to recombination in acinar, ductal, and islet cells in the pancreas, although Lenti-

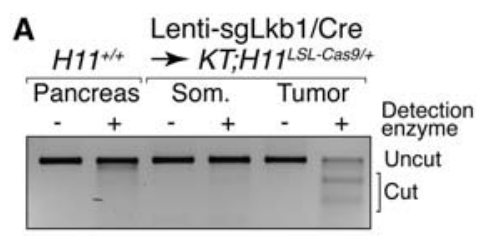

B

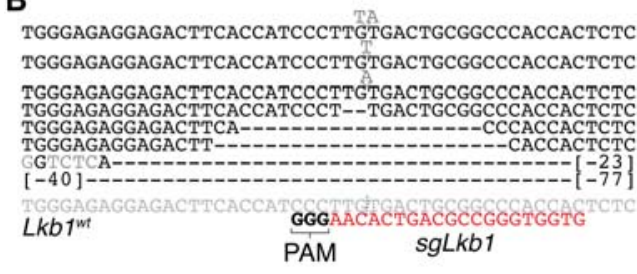

C

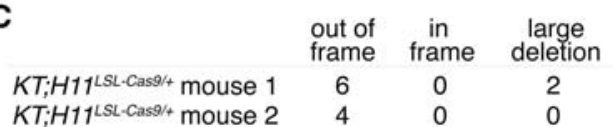

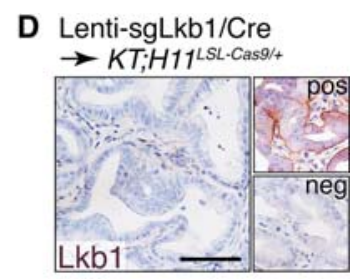

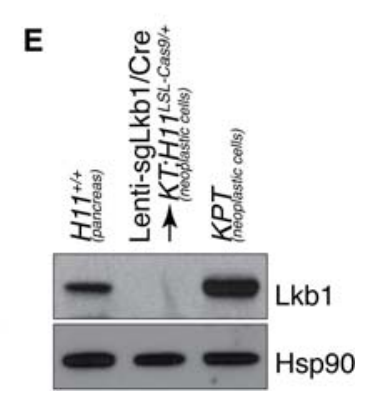

Figure 5. Cas9-mediated targeting leads to the formation of pancreatic tumors harboring deleterious Lkb1 mutations and lacking Lkb1 protein. (A) Genomic cleavage detection assay detected indels in the targeted $L k b 1$ locus in Tomato ${ }^{\text {positive }}$ cells isolated from Lenti-sgLkb1/Cre-infected KT;H11 ${ }^{\text {LSL-Cas } 9 /+}$ mice. (Som) Somatic cells. (B) Eight mutated Lkb1 alleles present in a single, oligoclonal, Tomato ${ }^{\text {positive }}$ tumor mass from a Lenti-sgLkb1/Cre-infected $K T$; H11 ${ }^{\text {LSL-Cas } /++}$ mouse. The targeted Lkb1 locus, sgLkb1 sequence, and protospacer adjacent motif (PAM) are shown. (C) All observed indels in oligoclonal tumor masses create frameshift mutations in $L k b 1$. (D) Tumors in Lenti-sgLkb1/Cre-infected $K T$; $H 11^{\text {LSL-Cas } /++}$ mice lack Lkb1 protein. Positive control (pos) PDAC from an Ad-Cre-infected KP mouse and negative control (neg) tumor derived from a Lenti-sgLkb1/Cre-infected $K T ; L k b 1^{\text {flox/flox }}$ mouse are shown. Bar, $50 \mu \mathrm{m}$. (E) Neoplastic cells from the pancreas of Lenti-sgLkb1/Cre-infected $K T$; H11 $1^{\text {LSL-Cas } 9 /+}$ mice lack Lkb1 protein. Hsp90 shows

equal loading. KPT pancreatic cancer cells and a whole $K T ; H 11^{+/+}$pancreas were positive controls. 
Cre had a very strong preference for acinar cells (Supplemental Fig. 4B). While a vast majority of pancreatic cancer studies have used non-cell type-specific promoters to drive Cre, models using transgenic and knock-in Cre(ER) alleles to enable cell type-specific expression of oncogenic Kras and tumor suppressor loss have been used to identify cells that possess the ability to give rise to PDAC /Guerra et al. 2007; Habbe et al. 2008; Gidekel Friedlander et al. 2009; Kopp et al. 2012; Puri et al. 2015). However, comprehensive studies that target diverse genomic alterations to a greater array of defined cell types are simply not feasible using current transgene-based systems. The use of adenoviral vectors with cell type-specific promoters has greatly accelerated the investigation of the cell of origin of lung adenocarcinoma and small cell lung cancer (Sutherland et al. $2011,2014)$, suggesting that similar approaches could rapidly advance our understanding of the precise cell type-genotype combinations that can give rise to PDAC.

Soft tissue sarcomas can be generated in $\operatorname{Kras}^{\text {LSL-G12D; }}$ $p 53^{f / f}$ mice by viral-Cre infection of cells within the muscle (Kirsch et al. 2007); therefore, we initially suspected that this would be a major impediment for generating viral-induced PDAC models. In fact, although retrograde ductal injection of Ad-Cre did induce extrapancreatic sarcomas in over half of the KPT mice, these were rarely problematic. PDAC initiation in KPT mice with an adenoviral vector containing the pancreatic-specific Ptf1a enhancer driving Cre expression solved this issue. We also found that many fewer Lenti-Cre-injected mice developed sarcomas, consistent with the limited ability of lentiviral vectors to induce sarcomas (Supplemental Table 1; DuPage et al. 2012). We envision that retrograde pancreatic ductal injection could become a ubiquitous technique for the generation of PDAC in adult mice. Importantly, the post-doctoral fellow who performed these surgeries (S.-H. Chiou) had no prior surgical experience and quickly mastered this technique. We experienced $\sim 10 \%$ mortality within the first week after surgery ( 19 out of 173 mice) and achieved nearly $100 \%$ successful injection as determined by the presence of numerous Tomato ${ }^{\text {positive }}$ cells in the pancreata of infected mice.

Integrating the CRISPR/Cas9 system into the pancreatic cancer biologists' toolkit via our viral and transgenic approaches should make loss-of-function experiments in pancreatic cancer in vivo no more difficult than altering those genes in vitro. This system should enable the rapid functional investigation of any gene of interest and allow genetic screens in pancreatic cancer in vivo. Investigating panels of putative tumor suppressor genes, factors that influence malignant progression, genes involved in cancer cell-stromal cell interactions, and genes that may modulate therapy responses could all be facilitated by this system. Conceptually, sgRNA-directed Cas9 cutting should also enable the rapid generation of structural genomic alterations observed in PDAC patients, and the ability to encode multiple sgRNAs on viral vectors will allow multiplexed gene inactivation in pancreatic cancer models (Maddalo et al. 2014; Platt et al. 2014).

Given the relative simplicity of generating pancreatic neoplasms and pancreatic cancer with these systems as well as the rapid and simple nature with which loss-offunction models can be generated using in vivo CRISPR/ Cas9 genome editing in the pancreas, we believe that this virally based PDAC model will provide new avenues to further understand the mechanistic basis of many aspects of pancreatic carcinogenesis.

\section{Materials and methods}

Mice

Kras $^{\text {LSL-G12D }}$, p53 $^{\text {flox }}, p 53^{L S L-R 172 H}, L k b 1^{\text {flox }}$, Rosa26 $6^{\text {Motley }}, C M V$ Cre, and Rosa26 $6^{\text {LSL-tdTomato }}$ (ai9), mice have been described (Schwenk et al. 1995; Jackson et al. 2001; Jonkers et al. 2001; Olive et al. 2004; Madisen et al. 2010; Nakada et al. 2010; Caswell et al. 2014). H11 ${ }^{\text {LSL-Cas } 9}$ site-specific knock-in mice (The Jackson Laboratory, stock no. 026816) were generated by micronuclear injection of a targeting plasmid and capped $\phi C 31$ mRNA into C57Bl/6 H11 ${ }^{\text {attPx3/attPx3 }}$ zygotes (Tasic et al. 2011) followed by implantation into pseudopregnant CD1 recipient mice. H11 Cas mice with constitutive Cas9 expression (The Jackson Laboratory, stock no. 027650) were generated by crossing H11 ${ }^{\text {LSL-Cas9 }}$ mice to CMV-Cre mice (The Jackson Laboratory, stock no. 006054). The Stanford Institutional Animal Care and Use Committees approved all animal studies and procedures.

\section{Tumor initiation and quantification}

Tumors were initiated by retrograde pancreatic ductal injection of mice with viral vectors as previously described (Supplemental Movie 1; Taniguchi et al. 2003). Briefly, laparotomy was performed on anesthetized mice, and half of the total bowel mass was gently pulled out of the abdomen, exposing the duodenum. A microclip was placed on the cystic duct close to the gallbladder, and a 30-gauge needle was inserted through the sphincter of Oddi into the common bile duct. One-hundred-fifty microliters of viral vector was injected over the course of 1-2 min. Subsequently, the microclip was removed, the duodenum and bowels were placed back into the abdomen, the muscle layer was sewn shut, and the skin was stapled with sterile suture clips. Once proficient, the entire procedure takes $<20 \mathrm{~min}$ per mouse.

\section{Generation of lentiviral vectors}

Ad-Cre and Ad-FLPo were purchased from the University of Iowa Gene Transfer Core. Lenti-PGK-Cre and Lenti-U6-sgRNA/PGKCre vectors were generated using standard methods and are available from Addgene (see the Supplemental Material for Addgene IDs). To assess the efficiency of genome editing with LentisgRNA/Cre vectors, we generated LSL-YFP cells that stably express Cas9 by infecting LSL-YFP cells with an MSCV-Cas9/ Puro vector followed by puromycin selection.

Histologic preparation, immunohistochemistry, and tumor analysis

Samples were fixed in $4 \%$ formalin and paraffin-embedded. Immunohistochemistry was performed on 4- $\mu$ m sections with the ABC Vectastain kits (Vector Laboratories) with antibodies to Tomato (Rockland Immunochemicals, 600-401-379), Muc5a (mouse monoclonal [45M1]; Abcam), CK19 (TROMA-III; Developmental Studies Hybridoma Bank), SMA (mouse monoclonal [1A4]; Sigma-Aldrich), and Lkb1 (Cell Signaling, 13031P). Sections were developed with $\mathrm{DAB}$ and counterstained with 
hematoxylin. Hematoxylin and eosin, Masson's trichrome, Alcian blue, and periodic acid-Schiff were performed using standard methods. All histological assessments were conducted by a board-certified pathologist (G.E. Kim).

\section{Western blotting}

Tail tip fibroblasts infected with viral-Cre and ex vivo tissue samples were homogenized and lysed with RIPA buffer. FACS-isolated cells were lysed with RIPA buffer. Denatured samples were run on a $4 \%-12 \%$ Bis-Tris gel (NuPage) and transferred onto PVDF membrane. Membranes were immunoblotted using primary antibodies against Hsp90 (BD Transduction Laboratories, 610419), Lkb1 (Cell Signaling, 13031P), actin (clone AC-15; Sigma-Aldrich, A1978), Cas9 (Novus Biologicals, NBP2-36440), Flag (Sigma-Aldrich, F1804), and secondary HRP-conjugated anti-mouse (Santa Cruz Biotechnology, sc-2005) and anti-rabbit (Santa Cruz Biotechnology, sc-2004) antibodies.

\section{Assessing CRISPR/Cas9-induced indels}

The GeneArt genomic cleavage detection (GCD) assay (Life Technologies) was used to analyze CRISPR/Cas9-mediated cleavage of the $L k b 1$ locus following the manufacturer's instructions. To further characterize the spectrum and frequency of Lkb1 mutations resulting from CRISPR/Cas9 targeting both in vitro and in vivo, $L k b 1$ amplicons were TOPO TA-cloned (Invitrogen), and $\sim 35$ clones from each sample were Sangersequenced.

\section{Acknowledgments}

We thank the Stanford Shared FACS Facility and the Protein and Nucleic Acid Facility for expert assistance; Carolyn Sinow for technical assistance; Jennifer Lin and Yanfeng Li for assistance generating the H11 ${ }^{\text {LSL-Cas } 9}$ mice; Sean Dolan for administrative support; and David Feldser, Julien Sage, the Winslow laboratory, and the Stanford pancreatic cancer research community for helpful comments. This work was supported by a Pancreas Cancer Action Network-American Association for Cancer Research (AACR) Award in memory of Skip Vinagh (13-20-25-WINS), National Institutes of Health (NIH) R01-CA175336, and NIH R21CA194910 to M.M.W; NIH K08-CA172676, a Pancreas Cancer Action Network-AACR Award, and the Sidney Kimmel Foundation for Cancer Research to D.R. Carpizo; and in part by a Stanford Cancer Institute support grant (P30-CA124435) from the National Cancer Institute. S.-H.C., C.-H.C., J.J.B., and B.M.G. were supported by Stanford Dean's Fellowships. C.-H.C. was additionally funded by an American Lung Association Fellowship. S.N. was supported by NIH R01-CA175336-S1. D.Y. was supported by a Stanford Graduate Fellowship. I.P.W. and D.R. Caswell were supported by National Science Foundation Graduate Research Fellowships. F.B.T. and D.R. Caswell were supported by NIH T32HG000044 and NIH T32CA09302, respectively. J.J.B. was additionally supported by NIH F32-CA189659. B.M.G. was additionally supported by a Pancreatic Cancer Action NetworkAACR Fellowship in memory of Samuel Stroum (14-40-25GRUE). S.K.K. is an investigator of the Howard Hughes Medical Institute. M.M.W. is a Donald E. and Delia B. Baxter Foundation Faculty Fellow and a V Foundation for Cancer Research Martin D. Abeloff, M.D., V Scholar. S.-H.C. conducted the surgical procedures and performed FACS and flow cytometry analyses, immunohistochemical staining, and dissecting scope imaging. I.P.W. contributed to $H 11^{\text {LSL-Cas9 }}$ allele generation and performed the functional validation, sgRNA design, and lentivirus generation and targeted DNA analysis. J.W. developed the retrograde ductal injection procedure. S.N. generated the H11 ${ }^{\text {LSL-Cas9 }}$ allele. C.D. and D.R. Carpizo generated the Ad-Ptf1a-Cre vector. D.Y., F.B.T., J.J.B., and C.-H.C. performed experiments. B.M.G. and S.K.K. provided conceptual advice. J.J.B. and D.R. Caswell provided critical reagents. H.Z. and P.C. provided technical assistance. G.E.K. performed histological analyses. M.M.W. supervised the research project. S.-H.C., I.P.W., and M.M.W. designed the project and wrote the manuscript with comments from all authors.

\section{References}

Aguirre AJ, Bardeesy N, Sinha M, Lopez L, Tuveson DA, Horner J, Redston MS, DePinho RA. 2003. Activated Kras and Ink4a/ Arf deficiency cooperate to produce metastatic pancreatic ductal adenocarcinoma. Genes Dev 17: 3112-3126.

Bardeesy N, Aguirre AJ, Chu GC, Cheng KH, Lopez LV, Hezel AF, Feng B, Brennan C, Weissleder R, Mahmood U, et al. 2006a. Both p16(Ink4a) and the p19(Arf)-p53 pathway constrain progression of pancreatic adenocarcinoma in the mouse. Proc Natl Acad Sci 103: 5947-5952.

Bardeesy N, Cheng KH, Berger JH, Chu GC, Pahler J, Olson P, Hezel AF, Horner J, Lauwers GY, Hanahan D, et al. 2006b. Smad4 is dispensable for normal pancreas development yet critical in progression and tumor biology of pancreas cancer. Genes Dev 20: 3130-3146.

Biankin AV, Waddell N, Kassahn KS, Gingras MC, Muthuswamy LB, Johns AL, Miller DK, Wilson PJ, Patch AM, Wu J, et al. 2012. Pancreatic cancer genomes reveal aberrations in axon guidance pathway genes. Nature 491: 399-405.

Boulay BR, Parepally M. 2014. Managing malignant biliary obstruction in pancreas cancer: choosing the appropriate strategy. World I Gastroenterol 20: 9345-9353.

Caswell DR, Chuang CH, Yang D, Chiou SH, Cheemalavagu S, Kim-Kiselak C, Connolly A, Winslow MM. 2014. Obligate progression precedes lung adenocarcinoma dissemination. Cancer Discov 4: 781-789.

Cho H, Herzka T, Zheng W, Qi J, Wilkinson JE, Bradner JE, Robinson BD, Castillo-Martin M, Cordon-Cardo C, Trotman LC. 2014. RapidCaP, a novel GEM model for metastatic prostate cancer analysis and therapy, reveals myc as a driver of Ptenmutant metastasis. Cancer Discov 4: 318-333.

DuPage M, Mazumdar C, Schmidt LM, Cheung AF, Jacks T. 2012. Expression of tumour-specific antigens underlies cancer immunoediting. Nature 482: 405-409.

Gidekel Friedlander SY, Chu GC, Snyder EL, Girnius N, Dibelius G, Crowley D, Vasile E, DePinho RA, Jacks T. 2009. Contextdependent transformation of adult pancreatic cells by oncogenic K-Ras. Cancer Cell 16: 379-389.

Guerra C, Schuhmacher AJ, Canamero M, Grippo PJ, Verdaguer L, Perez-Gallego L, Dubus P, Sandgren EP, Barbacid M. 2007. Chronic pancreatitis is essential for induction of pancreatic ductal adenocarcinoma by K-Ras oncogenes in adult mice. Cancer Cell 11: 291-302.

Habbe N, Shi G, Meguid RA, Fendrich V, Esni F, Chen H, Feldmann G, Stoffers DA, Konieczny SF, Leach SD, et al. 2008. Spontaneous induction of murine pancreatic intraepithelial neoplasia (mPanIN) by acinar cell targeting of oncogenic Kras in adult mice. Proc Natl Acad Sci 105: 18913-18918.

Hezel AF, Gurumurthy S, Granot Z, Swisa A, Chu GC, Bailey G, Dor Y, Bardeesy N, Depinho RA. 2008. Pancreatic LKB1 deletion leads to acinar polarity defects and cystic neoplasms. Mol Cell Biol 28: 2414-2425. 
Hingorani SR, Petricoin EF, Maitra A, Rajapakse V, King C, Jacobetz MA, Ross S, Conrads TP, Veenstra TD, Hitt BA, et al. 2003. Preinvasive and invasive ductal pancreatic cancer and its early detection in the mouse. Cancer Cell 4: 437-450.

Hingorani SR, Wang L, Multani AS, Combs C, Deramaudt TB, Hruban RH, Rustgi AK, Chang S, Tuveson DA. 2005. Trp53R172H and KrasG12D cooperate to promote chromosomal instability and widely metastatic pancreatic ductal adenocarcinoma in mice. Cancer Cell 7: 469-483.

Jackson EL, Willis N, Mercer K, Bronson RT, Crowley D, Montoya R, Jacks T, Tuveson DA. 2001. Analysis of lung tumor initiation and progression using conditional expression of oncogenic K-ras. Genes Dev 15: 3243-3248.

Jonkers J, Meuwissen R, van der Gulden H, Peterse H, van der Valk M, Berns A. 2001. Synergistic tumor suppressor activity of BRCA2 and p53 in a conditional mouse model for breast cancer. Nat Genet 29: 418-425.

Kirsch DG, Dinulescu DM, Miller JB, Grimm J, Santiago PM, Young NP, Nielsen GP, Quade BJ, Chaber CJ, Schultz CP, et al. 2007. A spatially and temporally restricted mouse model of soft tissue sarcoma. Nat Med 13: 992-997.

Kopp JL, von Figura G, Mayes E, Liu FF, Dubois CL, Morris JP, Pan FC, Akiyama H, Wright CV, Jensen $\mathrm{K}$, et al. 2012. Identification of Sox9-dependent acinar-to-ductal reprogramming as the principal mechanism for initiation of pancreatic ductal adenocarcinoma. Cancer Cell 22: 737-750.

Kruse EJ. 2010. Palliation in pancreatic cancer. Surg Clin North Am 90: 355-364.

Lo B, Strasser G, Sagolla M, Austin CD, Junttila M, Mellman I. 2012. Lkb1 regulates organogenesis and early oncogenesis along AMPK-dependent and -independent pathways. I Cell Biol 199: 1117-1130.

Maddalo D, Manchado E, Concepcion CP, Bonetti C, Vidigal JA, Han YC, Ogrodowski P, Crippa A, Rekhtman N, de Stanchina $\mathrm{E}$, et al. 2014. In vivo engineering of oncogenic chromosomal rearrangements with the CRISPR/Cas9 system. Nature 516: 423-427.

Madisen L, Zwingman TA, Sunkin SM, Oh SW, Zariwala HA, Gu H, Ng LL, Palmiter RD, Hawrylycz MJ, Jones AR, et al. 2010. A robust and high-throughput Cre reporting and characterization system for the whole mouse brain. Nat Neurosci 13: 133-140.

Morton JP, Jamieson NB, Karim SA, Athineos D, Ridgway RA, Nixon C, McKay CJ, Carter R, Brunton VG, Frame MC, et al. 2010. LKB1 haploinsufficiency cooperates with Kras to promote pancreatic cancer through suppression of p21-dependent growth arrest. Gastroenterology 139: 586-597.

Nakada D, Saunders TL, Morrison SJ. 2010. Lkb1 regulates cell cycle and energy metabolism in haematopoietic stem cells. Nature 468: 653-658.

Olive KP, Tuveson DA, Ruhe ZC, Yin B, Willis NA, Bronson RT, Crowley D, Jacks T. 2004. Mutant p53 gain of function in two mouse models of Li-Fraumeni syndrome. Cell 119: 847-860.

Platt RJ, Chen S, Zhou Y, Yim MJ, Swiech L, Kempton HR, Dahlman JE, Parnas O, Eisenhaure TM, Jovanovic M, et al. 2014. CRISPR-Cas9 knockin mice for genome editing and cancer modeling. Cell 159: 440-455.

Puri S, Folias AE, Hebrok M. 2015. Plasticity and dedifferentiation within the pancreas: development, homeostasis, and disease. Cell Stem Cell 16: 18-31.
Puzio-Kuter AM, Castillo-Martin M, Kinkade CW, Wang X, Shen TH, Matos T, Shen MM, Cordon-Cardo C, Abate-Shen C. 2009. Inactivation of p53 and Pten promotes invasive bladder cancer. Genes Dev 23: 675-680.

Rahib L, Smith BD, Aizenberg R, Rosenzweig AB, Fleshman JM, Matrisian LM. 2014. Projecting cancer incidence and deaths to 2030: the unexpected burden of thyroid, liver, and pancreas cancers in the United States. Cancer Res 74: 2913-2921.

Raimondi S, Lowenfels AB, Morselli-Labate AM, Maisonneuve P, Pezzilli R. 2010. Pancreatic cancer in chronic pancreatitis; aetiology, incidence, and early detection. Best Pract Res Clin Gastroenterol 24: 349-358.

Saborowski M, Saborowski A, Morris JP, Bosbach B, Dow LE, Pelletier J, Klimstra DS, Lowe SW. 2014. A modular and flexible ESC-based mouse model of pancreatic cancer. Genes Dev 28: 85-97.

Schwenk F, Baron U, Rajewsky K. 1995. A cre-transgenic mouse strain for the ubiquitous deletion of loxP-flanked gene segments including deletion in germ cells. Nucleic Acids Res 23: 5080-5081.

Su GH, Hruban RH, Bansal RK, Bova GS, Tang DJ, Shekher MC, Westerman AM, Entius MM, Goggins M, Yeo CJ, et al. 1999. Germline and somatic mutations of the STK11/LKB1 PeutzJeghers gene in pancreatic and biliary cancers. Am J Pathol 154: $1835-1840$.

Sutherland KD, Proost N, Brouns I, Adriaensen D, Song JY, Berns A. 2011. Cell of origin of small cell lung cancer: inactivation of Trp53 and Rb1 in distinct cell types of adult mouse lung. Cancer Cell 19: 754-764.

Sutherland KD, Song JY, Kwon MC, Proost N, Zevenhoven J, Berns A. 2014. Multiple cells-of-origin of mutant K-Ras-induced mouse lung adenocarcinoma. Proc Natl Acad Sci 111: 4952-4957.

Taniguchi H, Yamato E, Tashiro F, Ikegami H, Ogihara T, Miyazaki J. 2003. $\beta$-Cell neogenesis induced by adenovirus-mediated gene delivery of transcription factor pdx-1 into mouse pancreas. Gene Ther 10: 15-23.

Tasic B, Hippenmeyer S, Wang C, Gamboa M, Zong H, Chen-Tsai Y, Luo L. 2011. Site-specific integrase-mediated transgenesis in mice via pronuclear injection. Proc Natl Acad Sci 108: 7902-7907.

Waddell N, Pajic M, Patch AM, Chang DK, Kassahn KS, Bailey P, Johns AL, Miller D, Nones K, Quek K, et al. 2015. Whole genomes redefine the mutational landscape of pancreatic cancer. Nature 518: 495-501.

Wang Z, Zhu T, Rehman KK, Bertera S, Zhang J, Chen C, Papworth G, Watkins S, Trucco M, Robbins PD, et al. 2006. Widespread and stable pancreatic gene transfer by adeno-associated virus vectors via different routes. Diabetes 55: 875 884.

Whittle MC, Izeradjene K, Rani PG, Feng L, Carlson MA, DelGiorno KE, Wood LD, Goggins M, Hruban RH, Chang AE, et al. 2015. RUNX3 controls a metastatic switch in pancreatic ductal adenocarcinoma. Cell 161: 1345-1360.

Yachida S, Iacobuzio-Donahue CA. 2009. The pathology and genetics of metastatic pancreatic cancer. Arch Pathol Lab Med 133: 413-422. 


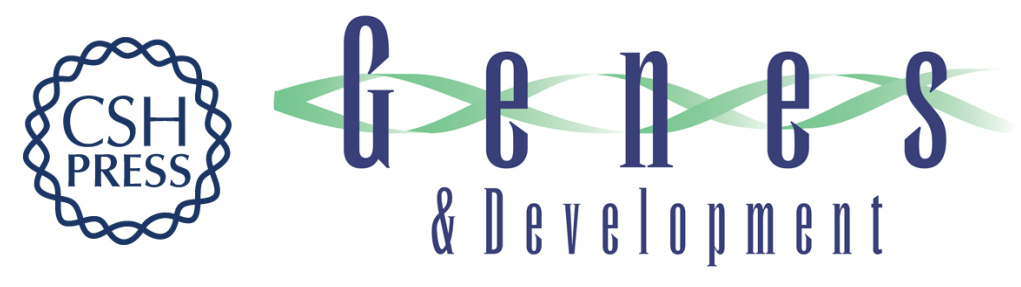

\title{
Pancreatic cancer modeling using retrograde viral vector delivery and in vivo CRISPR/Cas9-mediated somatic genome editing
}

\author{
Shin-Heng Chiou, lan P. Winters, Jing Wang, et al.
}

Genes Dev. 2015, 29: originally published online July 15, 2015

Access the most recent version at doi:10.1101/gad.264861.115

\section{Supplemental http://genesdev.cshlp.org/content/suppl/2015/07/13/gad.264861.115.DC1 Material}

References This article cites 41 articles, 15 of which can be accessed free at: http://genesdev.cshlp.org/content/29/14/1576.full.html\#ref-list-1

Creative This article is distributed exclusively by Cold Spring Harbor Laboratory Press for the first Commons six months after the full-issue publication date (see

License http://genesdev.cshlp.org/site/misc/terms.xhtml). After six months, it is available under a Creative Commons License (Attribution-NonCommercial 4.0 International), as described at http://creativecommons.org/licenses/by-nc/4.0/.

Email Alerting Receive free email alerts when new articles cite this article - sign up in the box at the top Service right corner of the article or click here.

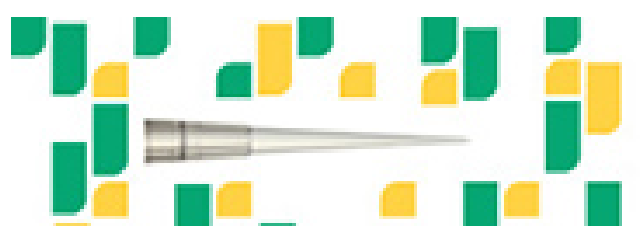

Focused on your science. 